\section{ITC 2/47}

Journal of Information Technology and Control

Vol. 47 / No. 2 / 2018

pp. 338-348

DOI 10.5755/j01.itc.47.2.19561

(c) Kaunas University of Technology

\section{Interpolating Bathymetric Big Data for an Inland Mobile} Navigation System

Received 2018/01/05

Accepted after revision 2018/04/23

\title{
Interpolating Bathymetric \\ Big Data for an Inland \\ Mobile Navigation System
}

\section{Marta Wlodarczyk-Sielicka}

Faculty of Navigation; Maritime University of Szczecin; Waly Chrobrego 1-2, 70-500 Szczecin, Poland, e-mail:m.wlodarczyk@am.szczecin.pl

\section{Natalia Wawrzyniak}

Marine Technology Ltd; Klonowica37/5, 71-248 Szczecin, Poland, e-mail: n.wawrzyniak@marinetechnology.pl

Corresponding author: m.wlodarczyk@am.szczecin.pl

Depth information is crucial in most navigational analysis and decision support implemented in existing inland navigation systems. Bathymetric data sets need to be preprocessed and converted into Digital Terrain Model (DTM) by interpolation methods to provide different vector layers for Electronic Navigational Chart. Data for inland waters need to be precise and valid due to quickly alternating inland environment and much shallower areas than on marine waters. At the same, time visual effect of created layers needs to be readable and easily interpreted by a navigator. In this paper, we analyse and assess the results obtained after using several interpolation methods for DTM building. The experiments used real inland data from bathymetric surveys conducted on the waters of Szczecin area. The main novelty of the research is the use of generally known interpolation algorithms during processing of bathymetric big data, which are the primary layer in new mobile system for inland navigation. The created depth contours are the base of navigational analysis provided by the mobile inland navigation system MOBINAV.

KEYWORDS: bathymetric data, interpolation method, maritime information systems, mobile systems.

\section{Introduction}

For many years, existing on-water navigation systems support marine and inland shipping and are essential for ensuring the safety of all traffic participants. In addition to providing spatial and navigational information of navigable area, some of these systems allow users for advance analysis that can help efficiently 
plan and safely conduct a voyage. There are both professional and standardized systems for commercial units, like ECDIS (Electronic Chart Display Information Systems) and simple mobile applications for recreational users as well $[16,17,18]$, which usually contain only an orthophotomap with smartphones localization feature in order to show ships geographical location. The MOBINAV system, which currently is being evolved by Marine Technology Ltd., is a compromise among professional solutions, their computational achievements and strict visualization standards with mobile technology and decision support. As a result, the final product incorporates basic objects of ECDIS systems with achievements of mobile cartography and user-centered design.

An absolute basis for all these systems is spatial information in a form of vector or raster map. The most important of all spatial information in such systems is depth information represented as vector layers for various types of analysis purposes. The majority of navigational alarms and warnings are generated using this information in combination with GPS position, current water-level information and draught of a vessel. Thus the base of correct analysis and navigational decision making is the possession of precise and actual depth information. However, the data not only have to be possibly detailed but the visual effect of presented spatial information must be readable, as not all the analysis that system offers are automatic. The majority of navigational decisions are made by a navigator himself or herself using only visual assessment of systems spatial data with reading measurements on board.

Raw bathymetric data are extremely large data sets and they need suitable spatial management and processing methods to be transformed into form that can be used by computer and especially mobile systems in a way to preserve its initial precision. Additionally, it is twice as difficult to assure its pleasant visual effect at the same time. That is why the problem of choosing the right interpolation methods for constructing a Digital Terrain Model (DTM) of underwater inland areas is not trivial.

In this article, we analyze different methods of bathymetric DTM interpolation to create depth contours in inland mobile navigation system. Taking into consideration the complex character of raw bathymetric data, we focus on finding a solution that generalizes data, but preserve shallowest information for the purpose of safety of navigation.

The paper is ordered as follows: Section 2 includes specification of the problems related to processing of spatial data comprehending depth information in mobile navigation systems; Section 3 describes a methodology of conducted analysis to choose the best method for DTM interpolation; Section 4 contains experimental results and the publication ends with an inference.

\section{Background and Related Work}

\subsection{The MOBINAV System}

The MOBINAV system is a semi-professional solution dedicated for inland recreational vessels which is being expanded actually by Marine Technology. MOBINAV takes advantage of spatial datasets combined with information provided by other navigational devices available on board, e.g. unit's positioning is the most important and can be obtained by a GPS (Global Positioning System) receiver of any kind. There is also a possibility of using other mobile device raw or derived sensors (magnetometer, barometer, ambient light) or attaching external ships sensors such as echosounder, AIS transponder, compass, etc. via WiFi. Any supplementary, non-spatial information, that assist in navigation can be obtained automatically from available online services, e.g., Web Map Services, River Information System, or to be filled in manually.

Generally, the MOBINAV system can be characterized by a semi-complex architecture that consists of two main parts - a server-side system and a mobile application that is divided into modules with deliberate interfaces to different sensors and external services. Server-side consists of three main modules of which two are strictly responsible for managing obtained static spatial data and preparing them into a chart that can be sent to users' mobile application upon chart update request. Third module manages dynamic spatial and nonspatial information provided and shared between users of MOBINAV. The mobile application has four major components. First two modules are responsible for storing locally acquired chart from the server side and visualizing its data in 
Figure 1

Simplified scheme of MOBINAV architecture based on [2]

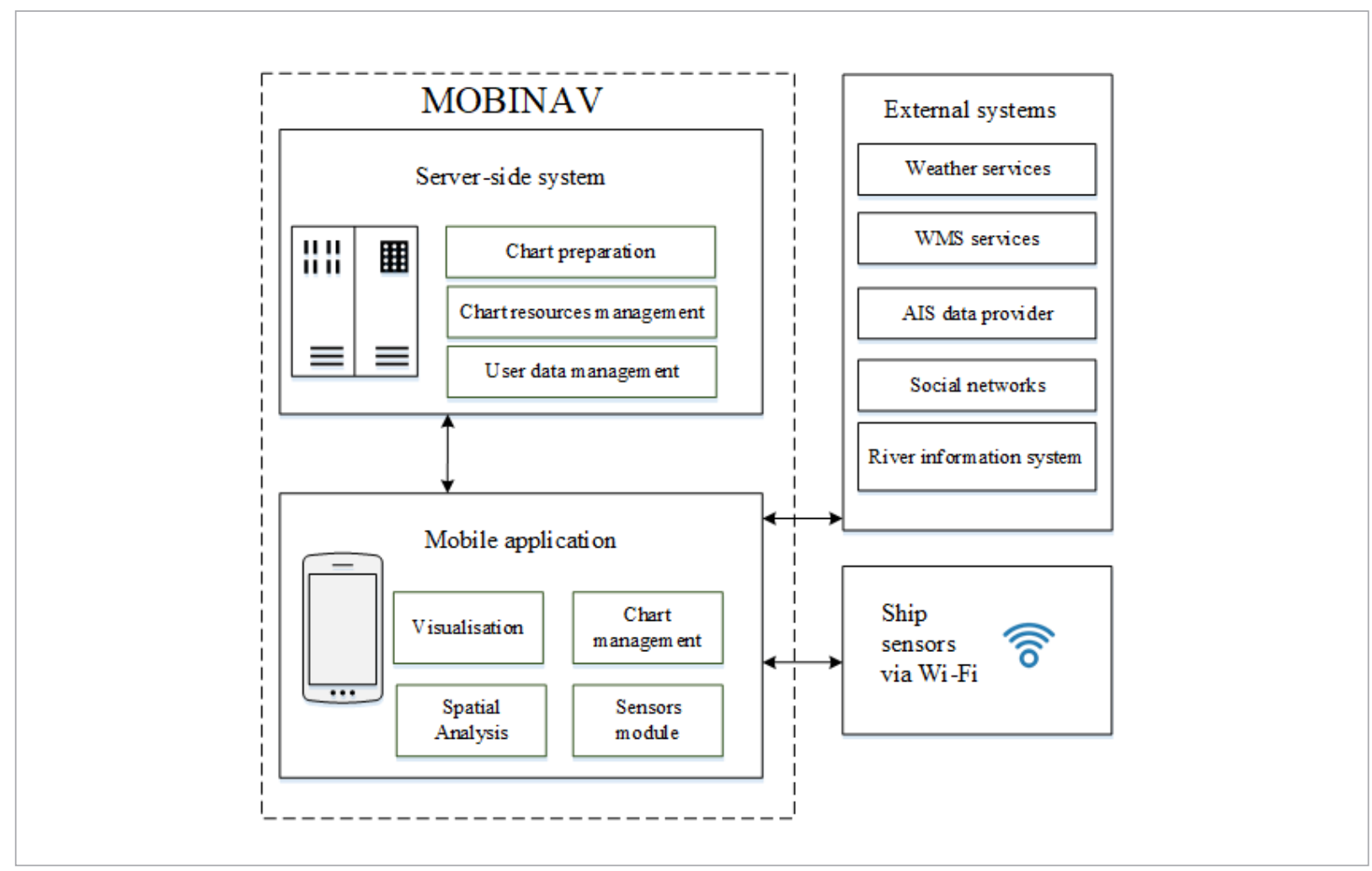

a display together with other navigational information that overlays the chart and may be acquired from different sources/services. Sensors module receives, processes and manages live data from internal and/or external sensors and allows spatial analyses module to conduct analyses based on acquired information together with spatial data and provides some decision support for a navigating user in form of alarms and warnings. An application must be in online mode to use external services as additional sources of both spatial and non-spatial information, e.g. the earlier mentioned WMS service or social networks. Such an information may be used to fulfill gaps or inaccuracies of charts and onboard sensors, but is treated separately by the system and cannot be the source of systems navigational analyses (Fig. 1).

The systems main spatial datasets that are transformed and integrated on the server side, fulfill dedicated MOBINAV Data Exchange Format (MODEF) model. The purposeful vector datasets for each spec- ified geographical area are then supplied to final users through XML/GML and converted into a map in the mobile application. Some raster datasets as an orthophotomap of chosen regions of particular interest (e.g., periodically complicated navigational areas, port, turning area) are also allowed [12]. Server side also makes it possible to share other important information, e.g., custom Point of Interest (POI), emergency information like Man Over Board (MOB) alarm.

The MOBINAV system is a kind of GIS (Geographic Information System) solution, which means that it is based on geometric vector data objects grouped in feature classes with a defined set of attributes. Neither objects geometry nor attributes that are grouped in core layers can be modified. However, new dynamic layers for spatial analyses purposes may be created and even shared among different users [5]. Moreover, all dynamic, information of ships traffic with appearing alerts and warnings that are created as an outcome of conducted analyses is represented as 
additional layers. The main analysis that uses depth information is proximity analysis for automatic alerting, when the ship is heading towards shallow areas. The system chooses safety isobaths based on vessels draught, water-level and the sets of depth area layer and indicates them on a map with red color (Fig. 2). The process systems constantly check if position of vessel and heading is not too close to indicated contour. If so, the system generates a warning and in closer proximity a danger alert.

The MOBINAV system has the visualization of data adjusted to the user's needs, with the purpose of using new mobile cartography. The main functional foundations of the MOBINAV were as follows [6]:

- import and integration of selected spatial data from public databases, according to the developed dedicated model of data;

_ implementation of spatial data analysis;

_ integration of internal and external navigation sensors;

_ visualization of spatial data according to the dedi- cated mobile cartographic presentation model;

- assistance of navigation by presenting the essential information;

_ implementation of routes management and route assistant;

_ management of own user data and personalization of application.

Designing of the MOBINAV system was, in fact, following methodologically conventional process of Geographic Information System project development with supplementary focus on mobile cartographic presentation. The workflow of the designing process included the following steps: analysis of users' requirements, specification, map data management, sensor data integration, spatial analysis, model of mobile cartographic presentation, generalization of spatial data and implementation of mobile technologies in the system.

As mentioned earlier, the fundamental of MOBINAV are spatial data from publicly available sources. The best source of data is Inland Electronic Navigational

\section{Figure 2}

Screenshots from MOBINAV installed on NOKIA1520: a) 2D view with active safe depth contour, b) $2+\mathrm{D}$ view with active safe depth contour, c) generated alarm for approaching shallow water area
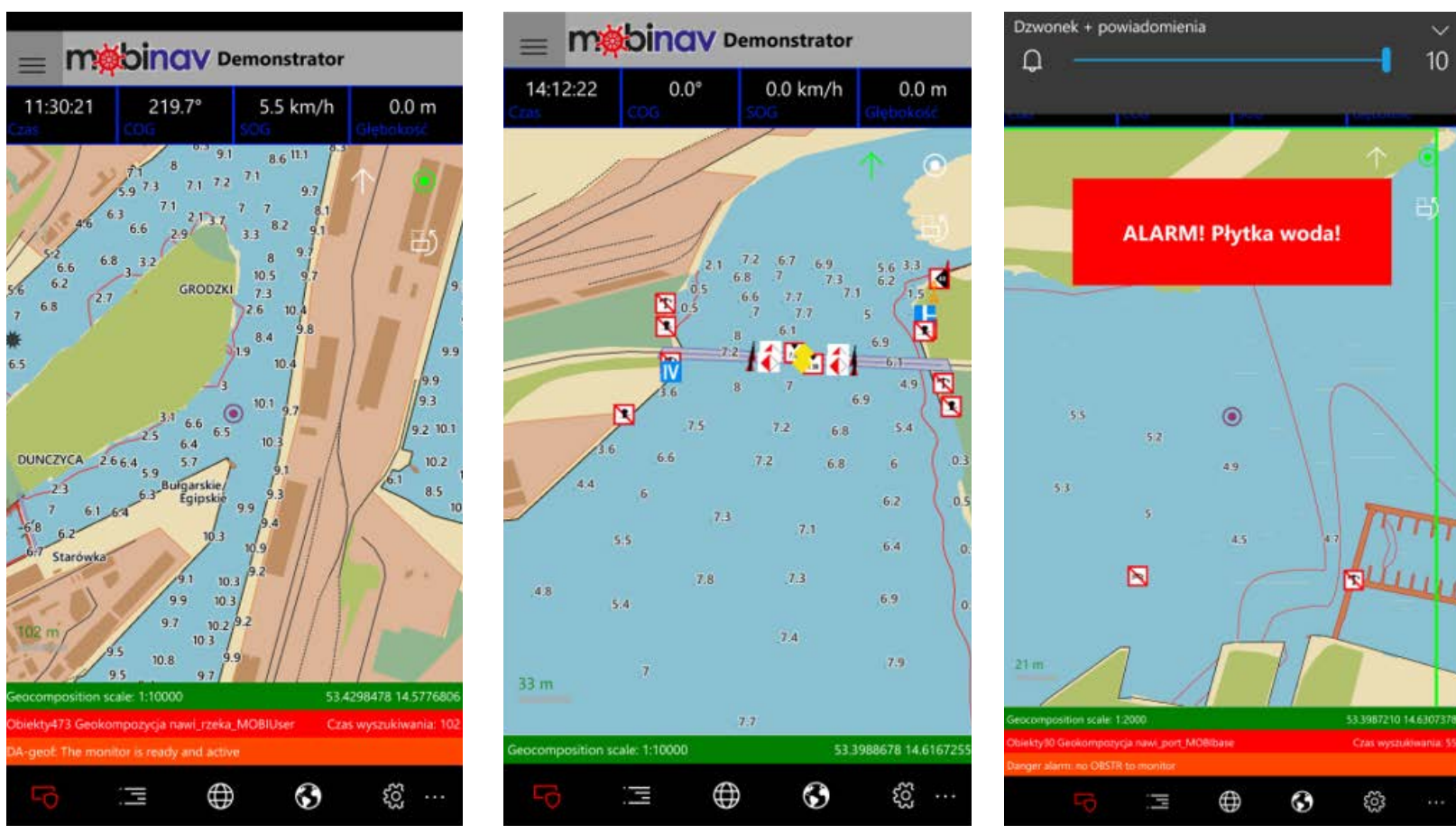
Figure 3

The process of data flow in the MOBINAV system

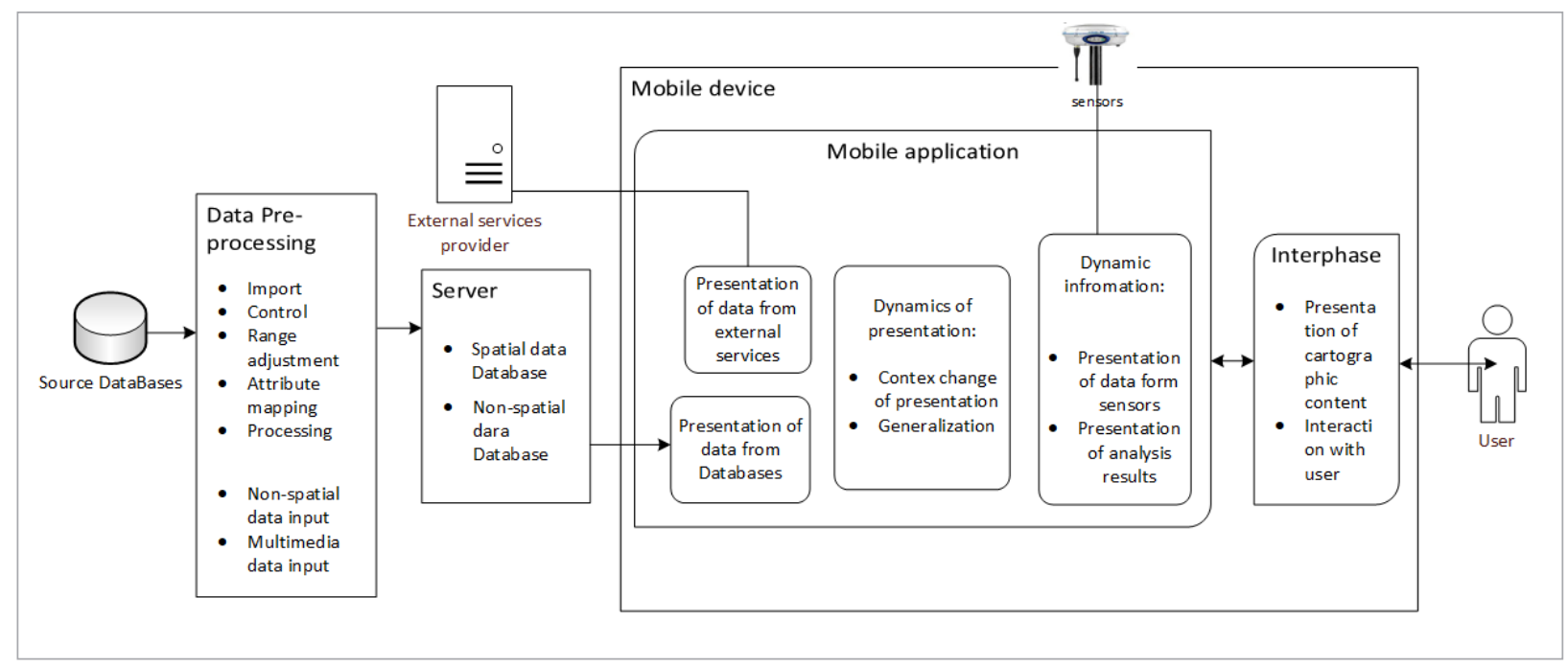

Chart IENC, which are common products in some areas. In order to guarantee data coverage in other waters, the use of other sources is indispensable. We decided to use the database of Vmap level 2, topographic data base BDOT10k, free data covered by open source project Open Street Map and data entered manually by the operator. The final result is geodatabase, from which a numerical map is created on the server and then sent to mobile devices. The dedicated spatial data model is defined as a UML class diagram according to the commonly used methodology. For its implementation, the rules for importing features from all source data are also determined. Elaboration of geodata in new format cover: importing source data to PostgreSQL/PostGIS database, processing and validation of geodata and export of final data to GML format. The process of data flow in the MOBINAV system is presented in Fig. 3.

The research subject is directly related to data generalization - the procedure that transforms and models spatial data for analytical and display purposes. It is very important step in each system that presents geographical data [10]. During creating the new system we focused on ensuring efficiency of cartographic communication. It was associated with the main goals of the project being created. The efficiency of cartographic communication has connection with three elements: the effectiveness of contents, serviceableness of numerical map and its usefulness to the end user [7]. On this basis we based the assessment of the results obtained in the research. The quantity of spatial data needed to load for the purpose of geovisualization is important. Given that the target equipment in the MOBINAV is a mobile device with small-display, it is necessary to execute a generalization of all layers.

\subsection{Bathymetric Data in an Inland Mobile Navigation System}

Bathymetry is the study and mapping of seabed topography and it involves obtaining measurements of the depth of water area. Bathymetric maps are mostly created to assistance safety of surface navigation and demonstrate seafloor as selected depths (soundings) and contour lines (called depth contours or isobaths). Charts also provide other navigational information. During the work on building of the mobile navigation system for inland waters it was decided to compile an own spatial data model MODEF (MObinav Data Exchange Format). Under the development of the model, 28 classes of geometric objects were defined, together with attributes describing them. The individual feature classes in the proposed model correspond to the information layers in the cartographic product, which is used to visualize data on the screen of the mobile device. In the system, there are two layers related with depth: soundings and depth areas. The first layer has point geometry and depth attribute at a given lo- 
cation. The second one has polygon geometry and two attributes: minimum and maximum depth. These layers are closely related to the aforementioned alarm, when the ship is heading towards shallow waters.

Depth areas or related with them depth contours can be obtained from a Digital Terrain Model DTM (e.g., a systematic grid of spots connected into a surface) [1, 11, 14]. To obtain such model, real bathymetric survey of mapped area need to be conducted. From the cloud of point measurements, one can build a DTM using either one of triangulation methods that preserves measured values in model vertices or proceed gridding algorithm that creates DTM in square GRID net with altered measurements in nodes but regularly structured, which facilitates later processing. On the other hand, benefit of using a TIN (Triangulated Irregular Network) over a GRID is that the points of a TIN are spread variably depending on an algorithm that determines which points are chosen for the purpose of accurate representation of the terrain. From the visual point of view, gridding algorithms allow to create models, which enhance proper users' reception, which in this case is important, as navigational decisions are mostly taken based on visual assessment of the situation, both in surroundings of the vessel and in the system display as well.

One cannot forget that bathymetric data, even more than any other type of spatial data present in GIS systems are burdened with certain, not negligible uncertainty. On one hand, it comes from obvious measurement limitation of real data gathering process with data post processing methods and on the other from used interpolation and generalization algorithms for the purpose of software performance and final visual effect. Bearing that fact in mind, users must be provided with most precise information to be able to undertake correct navigational decisions.

There are many gridding algorithms that can be considered in building a DTM but most of them cannot be used to build digital bathymetric model. It will provide the basis for later spatial analyses and decision support for inland navigation on mostly shallow waters and in quickly alternating environment.

Triangulation method is very precise because it holds real values in vertex point of DTM model but it might be resource-intensive, which in mobile systems is highly undesirable. On the other hand, it reproduces fast gradients and other sudden terrain changes very well [4].

Inverse distance weighted interpolation is commonly used and it makes the assumption that things that are close to one another are more alike than those that are farther apart. These methods are mostly global methods and are very simple to implement but theirs complexity is $\mathrm{O}(\mathrm{N})$ [9]. The equation of the method is shown below:

$$
z=\frac{\sum_{i=1}^{N} \frac{z_{i}}{\left(d_{i}+\sigma\right)^{p}}}{\sum_{i=1}^{N} \frac{1}{\left(d_{i}+\sigma\right)^{p}}},
$$

where: $z_{i}$ is the next value in measurement point, $d_{i}$ is the distance between a node and next measurement point, $p$ is the weighting power, $\sigma$ is the smoothing factor, $N$ is the number of interpolated points.

Modified Shepard's algorithm is also an inverse weighting algorithm but working locally (doesn't take into account measurements that are beyond set radius) [19].

The other commonly used method is Kriging (Gausian process regression) which is not a deterministic interpolation method like those mentioned above but gives the best linear unblast prediction of the intermediate values. The general formula is formed as a weighted sum of the data:

$Z\left(S_{0}\right)=\sum_{i=1}^{N} \lambda_{i} Z\left(S_{i}\right)$,

where: $Z\left(S_{i}\right)$ is the measured value at the $i$ location

$\lambda_{i}$ is an unknown weight for the measured value at the i location, $S_{0}$ is the prediction location, $N$ is the number of measured points.

Weight computation is based on the inverted variogram. Kriging is most appropriate when one knows there is a spatially correlated distance or directional bias in the data [8]. It is often used in soil science and geology.

There is also the nearest neighbor method but besides its simplicity and the fact of filling existing gaps in survived data it does not build visually realistic DTM [3]. The main advantage of natural neighbor methods is that it does not extrapolate the model that is built, but that problem does not influence data in the MOBINAV system, because water depth data layer is al- 
ways limited by land area layer.

The other group of modeling DTM methods are based on radial functions - the most commonly used is a multiquadratic function, which is one of the most precise methods of interpolation dependent on smoothing parameter, but it is not efficient enough for precise bathymetric data. All neural networks interpolation methods are too complex for use in a mobile system.

\section{The Experiment}

All the test methods were implemented using Surface software, developed by Golden Software. Additionally, for visualization purposes, ArcGIS software was used. To gather bathymetric datasets the vessel $\mathrm{Hy}-$ drograf XXI, with interferometric GeoSwath Plus 250 $\mathrm{kHz}$ echosounder and extra equipment such as satellite compass, GPS/RTK receiver and motion sensor installed, was used.

\subsection{Test Area}

Test datasets were collected within Port of Szczecin, on the Babina Canal which is shown in Fig. 4.

\section{Figure 4}

Test area

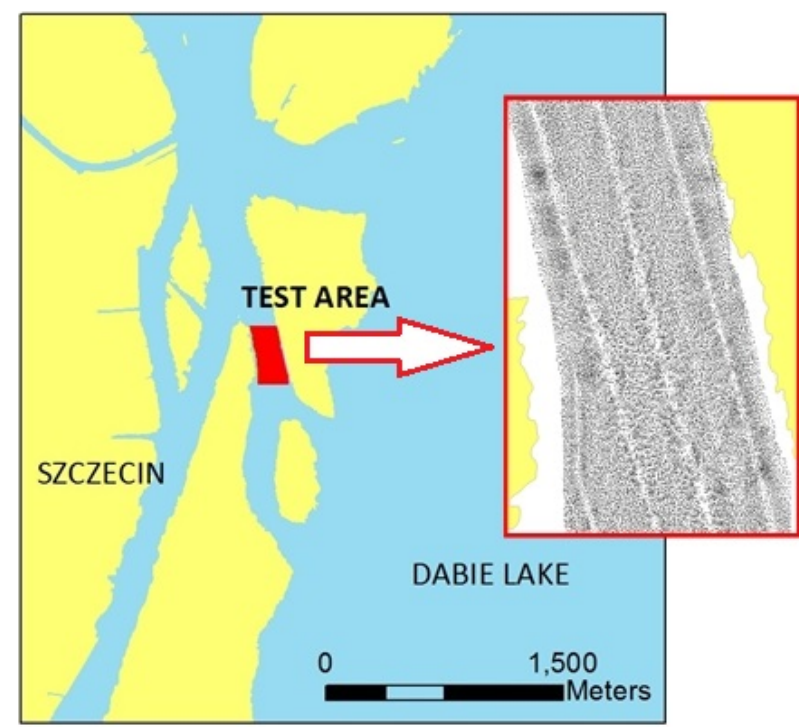

Raw bathymetric data are of very-high density and consist of XYZ points (latitude, longitude and depth). During the hydrographic exploration a very large amount of data was collected. The raw test dataset included 5864171 points of XYZ elements. Each sample has three attributes: latitude, longitude, and a predetermined true depth at a given position. A large raw dataset is the primary operational restriction when using a regular computer. In order to solve this problem, we decided to use smaller subsets - data after reduction. For the purposes of this article, a reduced dataset was created using the method developed by one of the authors - for a scale of 1: 500. A part of this method is presented in $[13,15]$.

The dataset after reduction included $25843 \mathrm{XYZ}$ points and it is shown in Fig. 2. The minimum value of depth is $0.3 \mathrm{~m}$, the maximum value of depth is $8.96 \mathrm{~m}$ and the mean value of depth is $6.08 \mathrm{~m}$.

\subsection{Research Methodology}

For publication purposes, we have chosen four different gridding interpolation methods that best represented different interpolation approach in DTM creation, but at the same time, are computationally possible to process on big sets of bathymetric data: triangulation with linear interpolation, Kriging, the modified Shepard's method and inverse distance to a power. The first step involved the development of grid for test dataset using selected method. It was implemented using Surface software with the following parameters:

_ Triangulation with Linear Interpolation in which anisotropy ratio equals to 1 and anisotropy angle equals to 0 .

- Kriging in which polynomial drift order equals 0 and semi-variogram model has the following characteristics: linear component type and anisotropy angle equal to 0 . Additionally, anisotropy ratio equal to 1 , variogram slope equal to 1 and search parameters in which search ellipse radius 1 equals to 220, search ellipse radius 2 equals to 220 , search ellipse angle equals to 0 , number of search sectors equal to 4 , maximum data per sector equal to 16 , maximum empty sectors equal to 3 , minimum data equal to 8 and maximum data equal to 64 .

- The modified Shepard's Method in which smoothing factor equals to 0 , quadratic neighbors equals to 13 , weighting neighbors equals to 19 , search ellipse radius 1 equals to 44 , search ellipse radius 2 equals to 44 , search ellipse angle equals to 0.

_ Inverse Distance to a Power in which weighting 
power equals to 2 , smoothing factor equals to 0 , anisotropy ratio equals to 1 and anisotropy angle equals to 0 . The following parameters have been set for the search: search ellipse radius 1 equal to 220 , search ellipse radius 2 equal to 220 , search ellipse angle equal to 0 , number of search sectors equal to 4, maximum data per sector equal to 16 , maximum empty sectors equal to 3 , minimum data equal to 8 and maximum data equal to 64 .

The next step was creation of contour lines for each grid where the default settings were applied. The intervals have been set at $0.5 \mathrm{~m}$. The received surfaces and isobaths have been visualized. For all sets of depth contours, the following statistics were calculated: the total number of isobaths, minimum depth, maximum depth, mean depth and standard deviation. After that, the volume to zero level was computed. In the next step, the errors between XYZ point (test data) and model surface were calculated - the differences between the height of test data and the height of the grid file. The differences are negative and positive, so it is necessary to calculate the absolute value in order to correctly calculate the error. In the last step, using statistical functions, the mean, maximum and minimum error values were calculated.

\section{Results}

As previously mentioned, during the research, we focused on efficiency of cartographic communication. Furthermore, the quantity of bathymetric geodata needed to load for the purpose of geovisualization and their accuracy is also very important.

After the implementation of the assumed parameters for each method, we obtained four surfaces, which are presented in Fig. 5. As one can see, each surface has a different depth range. The shallower areas are shown in a darker shade of blue, as is the case in the navigation map.

Figure 5

Surfaces obtained using gridding methods: a) Triangulation with Linear Interpolation, b) Kriging, c) Modified Shepard's Method, d) Inverse Distance to a Power

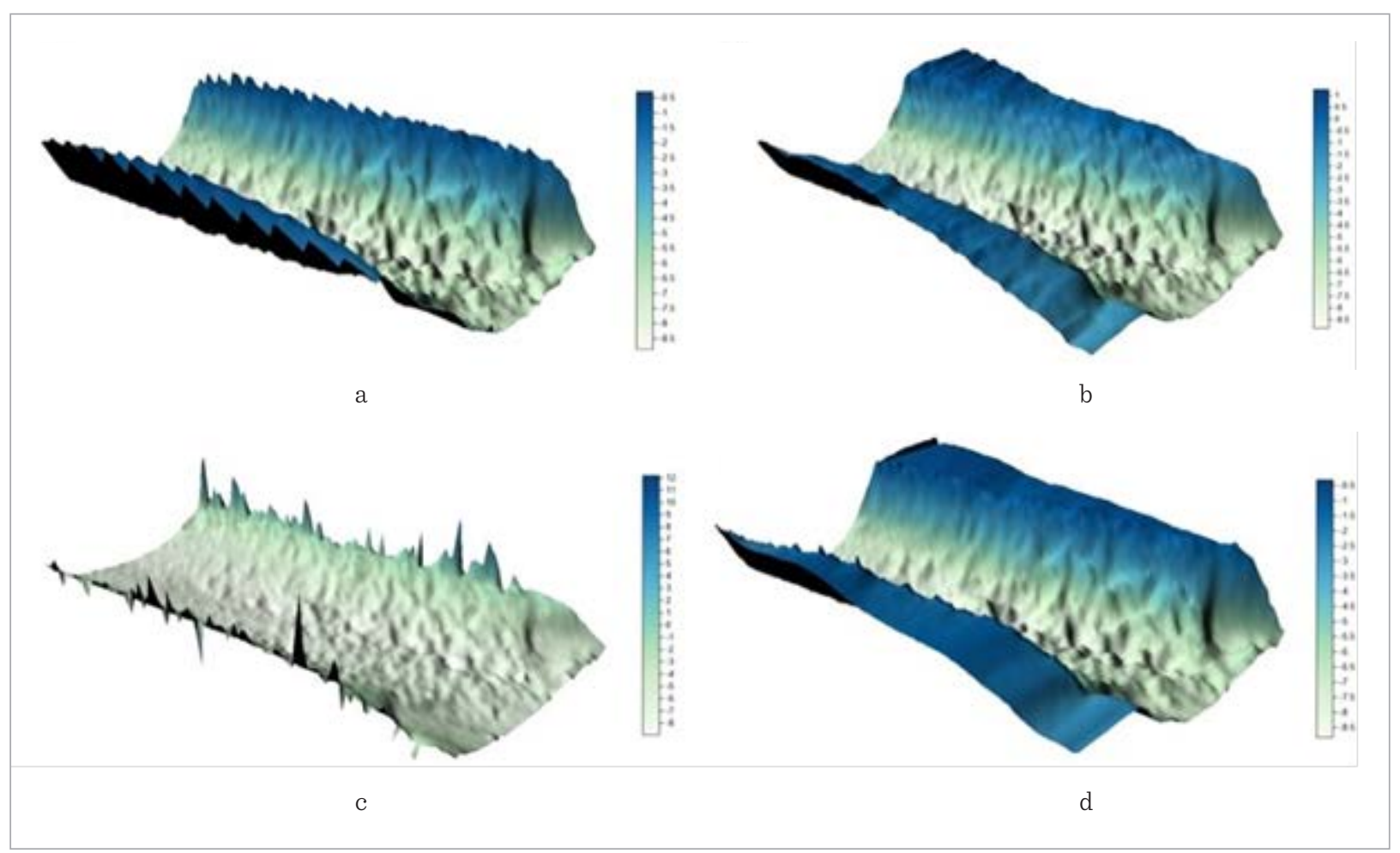


The surface that is most different from the rest is the surface obtained with the modified Shepard's gridding method. For each surface, volume to zero level was computed. For triangulation with linear interpolation method, the smallest volume was received and it equals to $381429.55 \mathrm{~m}^{3}$. For the modified Shepard's method, volume equal to $382457.91 \mathrm{~m}^{3}$ and, for Kriging volume equal to $391701.77 \mathrm{~m} 3$ were obtained. For inverse distance to a power method the biggest volume was obtained and it equals to $416392.95 \mathrm{~m}^{3}$.

In the next step, for each of the four surfaces, contour lines with $0.5 \mathrm{~m}$ intervals were generated. They were visualized using ArcGIS software and they are presented in Fig. 6.

\section{Figure 6}

Depth contour obtained using gridding methods: a) Triangulation with Linear Interpolation, b) Kriging, c) Modified Shepard's Method, d) Inverse Distance to a Power

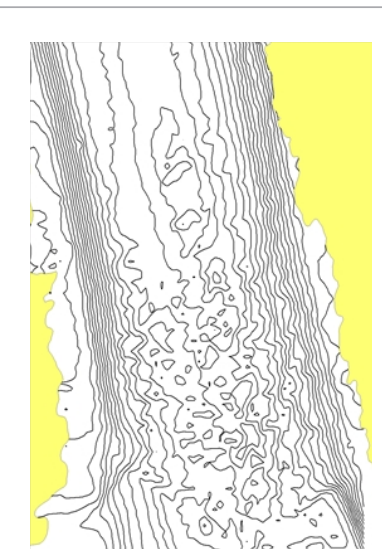

a

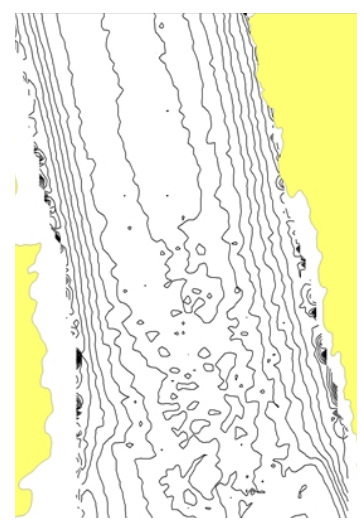

c

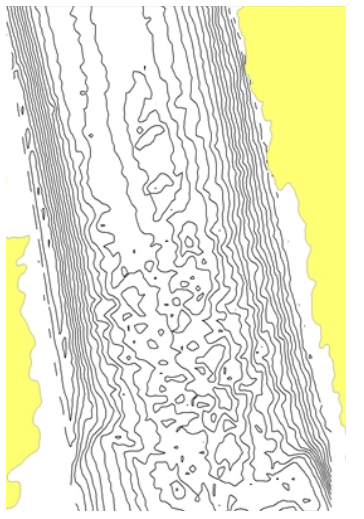

b

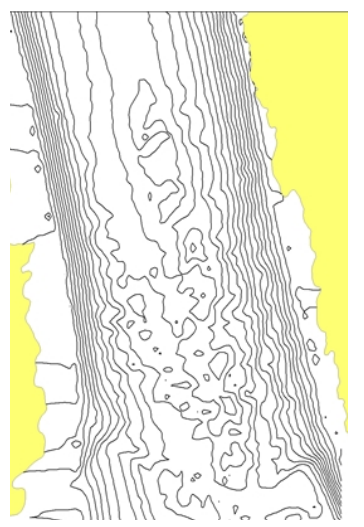

d
One can also notice that the isobaths obtained with the modified Shepard's gridding method are significantly out of place. The most similar are the isobaths obtained with triangulation with linear interpolation and Kriging methods. It is important to note that only isobaths obtained with triangulation with linear interpolation do not go beyond the coverage area of the source data. Table 1 contains statistics for all sets of depth contours.

\section{Table 1}

Statistics for all sets of depth contours

\begin{tabular}{l|c|c|c|c|c}
\hline \multicolumn{1}{|c|}{$\begin{array}{l}\text { Gridding } \\
\text { Methods }\end{array}$} & $\begin{array}{c}\text { Total } \\
\text { Num- } \\
\text { ber }\end{array}$ & $\begin{array}{c}\text { Min } \\
\text { Depth } \\
{[\mathrm{m}]}\end{array}$ & $\begin{array}{c}\text { Max } \\
\text { Depth } \\
{[\mathrm{m}]}\end{array}$ & $\begin{array}{c}\text { Mean } \\
\text { Depth } \\
{[\mathrm{m}]}\end{array}$ & $\sigma$ \\
\hline $\begin{array}{l}\text { Triangulation } \\
\text { (L.I.) }\end{array}$ & 155 & 0.50 & 8.50 & 4.92 & 2.83 \\
\hline Kriging & 121 & 0.00 & 8.50 & 5.60 & 2.60 \\
\hline $\begin{array}{l}\text { Modified } \\
\text { Shepard's }\end{array}$ & 326 & -12.00 & 8.00 & 1.11 & 4.38 \\
\hline $\begin{array}{l}\text { Inv. Dist. to } \\
\text { Power }\end{array}$ & 90 & 0.50 & 8.50 & 4.76 & 2.73 \\
\hline
\end{tabular}

All methods except Modified Shepard's method provided depth contours in realistic range, in which real values were measured. However, errors in the modified Shepard's method occur only on edges of created model and can be easily eliminated. Additionally, depth contours for tested methods were presented in one map. This can help in visual assessment of received results. An example of this map is shown in Fig. 7 at a 1:250 scale.

Fig. 7 indicates the differences between contour lines in the chart, generated at a 1:250 scale. The observed differences refer to few meters (below $3 \mathrm{~m}$ ) in both directions, which does not have crucial influence on navigational analyses. The warnings and alarms are generated in distance at least of $100,200 \mathrm{~m}$ from obstacles or shallow waters, to give a navigator time to react.

Table 2 reports the errors between the height at the test XYZ sample and the height calculated in the grid file.

All the tested methods provided minimum error equal to zero. The mean errors are similar: between $0.08 \mathrm{~m}$ 
Figure 7

Depth contours obtained using gridding methods at a 1:250 scale

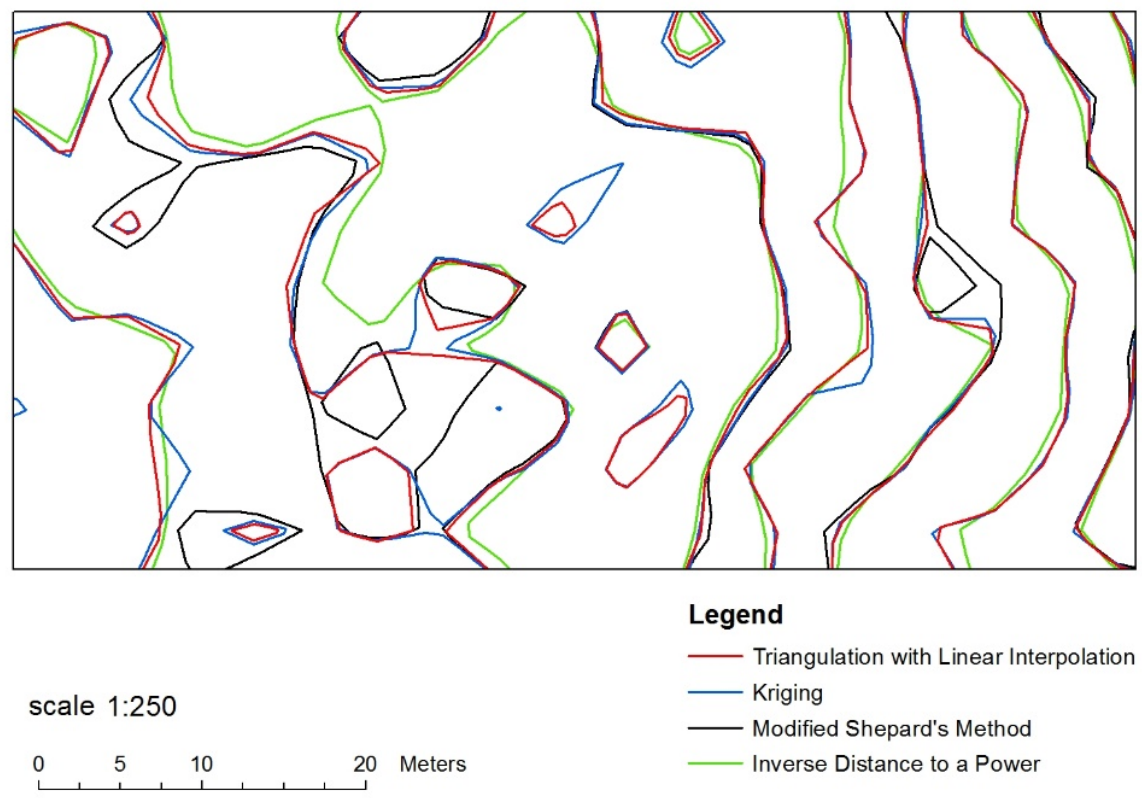

Table 2

The errors between test data and model surfaces

\begin{tabular}{l|c|c}
\multicolumn{1}{c|}{ Gridding Methods } & Mean Error [m] & Max Error [m] \\
\hline Triangulation (L.I.) & 0.08 & 1.05 \\
\hline Kriging & 0.08 & 2.04 \\
\hline Modified Shepard's & 0.08 & 1.94 \\
\hline Inv. Dist. to Power & 0.09 & 2.15
\end{tabular}

and $0.09 \mathrm{~m}$. We received the smallest error for triangulation method.

\section{Conclusions}

The main novelty of the research is the use of generally known interpolation algorithms during processing of bathymetric big data, which are primary layer in new mobile system for inland navigation. Visual communication of cartographic displays, especially small-display of mobile devices, requires that the user attend to and extract all important information from a display during visual exploration. The MOBINAV system is an innovative solution for inland recreational navigation. Users of the created system should be able to correctly interpret the mapped message. We checked the use of known interpolation methods for bathymetric big data. We focused mainly on getting the data legible and accurate.

In the experiment, after implementing each analyzed method, we obtained different results which we presented in the form of a surface model. Therefore, we generated depth contours from each of them. From tested methods, the model generated by triangulation has the most advantages. The first to mention is that, the obtained model keeps real measurement points, as it does not include gridding algorithm. Generated isobaths do not exceed surveyed area and their shape itself closely resembles the model obtained using the Kriging method.

For the safety of navigation reasons, it is essential to preserve the information of shallowest areas. Triangulation method with linear interpolation provides unchanged values in model vertices, so assuming the survey was conducted properly - the model provides the most reliable information. 


\section{References}

1. Amante, C. J., Eakins, B. W. Accuracy of Interpolated Bathymetry in Digital Elevation Models Journal of Coastal Research. 2016, Special Issue: 76, 123-133. https://doi.org/10.2112/SI76-011

2. Baus, J., Krüger, A. and Wahlster, W. A Resource-Adaptive Mobile Navigation System. In Proceedings of 7th International Conference on Intelligent User Interfaces, IUI '02, New York, USA, 2002, 15-22. ACM. https:// doi.org/10.1145/502716.502723

3. Greenspan, M., Godin G. A Nearest Neighbor Method for Efficient ICP. In Proceedings of the 3rd IEEE International Conference on 3-D Digital Imaging and Modeling, Quebec, 2001, 161-168. https://doi.org/10.1109/ IM.2001.924426

4. Fowler, R. J., Little, J. J. Automatic Extraction of Irregular Network Digital Terrain Models. ACM SIGGRAPH Computer Graphics, 1979, 13(2), 199-20\%. https://doi. org/10.1145/965103.807444

5. Kazimierski, W., Wlodarczyk-Sielicka, M. Technology of Spatial Data Geometrical Simplification in Maritime Mobile Information System for Coastal Waters. Polish Maritime Research, 2016, 3(23), 3-12, Gdansk University of Technology, ISSN 1233-2585

6. Kazimierski, W., Zaniewicz, G., Bodus-Olkowska, I. Integrated Presentation of Navigational Data in a Mobile Navigation System for Inland Waters with the Use of HUD. Scientific Journal of Maritime University of Szczecin, 2017, 49(121), 84-92, DOI: 10.17402/203

7. Kraak, M.-J. Cartographic Design. The International Encyclopedia of Geography, 2017, 1-16. https://doi. org/10.1002/9781118786352.wbieg0603

8. Li, J., Heap, A. D. A Review of Spatial Interpolation Methods for Environmental Scientists. Geoscience Australia: Canberra, Australia, 2008. https://doi. org/10.1016/j.envsoft.2013.12.008

9. Lu, G. Y., Wong, D. W. An Adaptive Inverse-Distance Weighting Spatial Interpolation Technique. Computers \& Geosciences, 2008, 34(9), 1044-1055. https://doi. org/10.1016/j.cageo.2007.07.010

10. McMaster, R. B., Shea, K. S. Generalization in Cartography. Association of American Geographers, Washington, D. C., 1992.

11. Shimada, K., Aoki, S., Ohshima, K. I. Creation of a Grid- ded Dataset for the Southern Ocean with a Topographic Constraint Scheme. Journal of Atmospheric and Oceanic Technology, 2017, 34(3), 511-532. https://doi. org/10.1175/JTECH-D-16-0075.1

12. Wawrzyniak, N., Hyla, T. Application of Geofencing Technology for the Purpose of Spatial Analyses in Inland Mobile Navigation. Proceedings of Baltic Geodetic Congress (Geomatics), Gdansk University of Technology, 2016. https://doi.org/10.1109/BGC.Geomatics.2016.15

13. Wlodarczyk-Sielicka, M. Stateczny, A. Fragmentation of Hydrographic Big Data into Subsets During Reduction Process. Proceedings of Baltic Geodetic Congress (Geomatics), Gdansk University of Technology, 2017. https://doi.org/10.1109/BGC.Geomatics.2017.67

14. Wlodarczyk-Sielicka, M., Stateczny, A. Comparison of Selected Reduction Methods of Bathymetric Data Obtained by Multibeam Echosounder. Proceedings of Baltic Geodetic Congress (Geomatics), Gdansk University of Technology, 2016. https://doi.org/10.1109/BGC.Geomatics.2016.22

15. Wlodarczyk-Sielicka, M. Importance of Neighborhood Parameters During Clustering of Bathymetric Data Using Neural Network. Communications in Computer and Information Science, 2016, 639, 441-452, ISSN 18650929. https://doi.org/10.1007/978-3-319-46254-7_3

16. Wozniak, M. Novel Image Correction Method Based on Swarm Intelligence Approach. Communications in Computer and Information Science, 2016, 639, 404413, ISSN 1865-0929. https://doi.org/10.1007/978-3319-46254-7_-32

17. Wozniak, M., Polap, D., Napoli, C., Tramontana, E. Graphic Object Feature Extraction System Based on Cuckoo Search Algorithm. Expert Systems with Applications, 2016, 66, 20-3. https://doi.org/10.1016/j. eswa.2016.08.068

18. Wozniak, M., Polap, D. Hybrid Neuro-Heuristic Methodology for Simulation and Control of Dynamic Systems over Time Interval. Neural Networks, 2017, 93, 4556. https://doi.org/10.1016/j.neunet.2017.04.013

19. Zuppa, C., Error Estimates for Modified Local Shepard's Interpolation Formula. Applied Numerical Mathematics, 2004, 49, 245-259. https://doi.org/10.1016/j. apnum.2003.11.001 